\title{
ISSN 2767-3901 High Order Godunov Type Multimesh Method for 3d Impact Problems of Elastoplastic Media
}

International Journal of Theoretical \& Computational Physics

\author{
Research Article
}

\author{
M.H.Abuziarov \\ M.H.Abuziarov \\ Lobachevsky University, N.Novgorod, 23 Gagarin Ave., \\ Russia
}

*Correspondence author

\author{
M.H.Abuziarov \\ Lobachevsky University \\ N.Novgorod, \\ 23 Gagarin Ave., \\ Russia
}

Submitted : 4 Jun 2021 ; Published : 23 Jun 2021

\begin{abstract}
A numerical method for calculating the three-dimensional processes of impact interaction of elastoplastic bodies under large displacements and deformations based on the multi mesh sharp interface method and modified Godunov scheme is presented. To integrate the equations of dynamics of an elastoplastic medium, the principle of splitting in space and in physical processes is used. The solutions of the Riemann problem for first and second order accuracy for compact stencil for an elastic medium in the case of an arbitrary stress state are obtained and presented, which are used at the "predictor" step of the Godunov scheme. A modification of the scheme is described that allows one to obtain solutions in smoothness domains with a second order of accuracy on a compact stencil for moving Eulerian-Lagrangian grids. Modification is performed by converging the areas of influence of the differential and difference problems for the Riemann's solver. The "corrector" step remains unchanged for both the first and second order accuracy schemes. Three types of difference grids are used. The first - a moving surface grid-consists of a continuous set of triangles that limit and accompany the movement of bodies; the size and number of triangles in the process of deformation and movement of the body can change. The second - a regular fixed Eulerian gridis limited to a surface grid; separately built for each body; integration of equations takes place on this grid; the number of cells in this grid can change as the body moves. The third grid is a set of local Eulerian-Lagrangian grids attached to each moving triangle of the surface from the side of the bodies and allowing obtain the parameters on the boundary and contact surfaces. The values of the underdetermined parameters in cell's centers near the contact boundaries on all types of grids are interpolated. Comparison of the obtained solutions with the known solutions by the Eulerian-Lagrangian and Lagrangian methods, as well as with experimental data, shows the efficiency and sufficient accuracy of the presented three-dimensional methodology.
\end{abstract}

Keywords: Elastoplastic Medium, Modeling, Eulerian-Lagrangian Approach, Godunov Scheme, Riemann Problem, Multi Mesh Algorithm, Interpolation, high-speed mpact.

Introduction

Godunov's scheme $[1,2]$, based on the use of the exact solution of the Riemann problem for integrating conservation laws when modeling fast processes in gas dynamics, allows the use of Lagrangian, Euler-Lagrangian, and Euler approaches. The popularity of the scheme is largely due to the use of the exact solution of the Riemann problem, which makes it possible to isolate shock fronts and contact discontinuities and attach mobile computational grids to them. In addition, the scheme is monotonic on discontinuous solutions. Numerous modifications of the scheme are known for modeling various physical processes in elastic [3-5], elastoplastic [6-8] and multiphase $[9,10]$ media. The main drawback of the Godunov scheme is the significant unregulated numerical viscosity due to the first order approximation. In this regard, attempts have been made to improve the accuracy of the scheme in various ways. For the problems of gas dynamics, these are modifications [11-15], in which an increase in accuracy is achieved by increasing the difference stencil. In [16-18], a method for increasing the accuracy of Godunov's scheme, which does not require an in- crease in the difference stencil when using Lagrangian and Euler-Lagrangian approaches, was presented. An increase in the approximation order to the second is achieved by converging the areas of influence of the differential and difference problems for an irregular moving grid. Approaches to improving the accuracy of the Godunov scheme for solving elastoplastic problems is described in [19-22]. Almost all of the above results in solving elastoplastic problems were obtained in the two-dimensional approximation.

In this paper, a modification of Godunov's scheme of increased accuracy for compact stencil for solving three-dimensional dynamic elastoplastic problems is described.

\section{Mathematical statement of the problem}

The system of equations describing the adiabatic unsteady elastoplastic deformation of a continuous medium in the absence of viscosity and thermal conductivity in the Euler Cartesian coordinate system Oxyz has the following form: 
$\frac{\partial}{\partial t} \mathbf{u}+\frac{\partial}{\partial x} \mathbf{f}+\frac{\partial}{\partial y} \mathbf{g}+\frac{\partial}{\partial z} \mathbf{h}=\mathbf{k}$

Where $\mathbf{u}=\left[\begin{array}{c}\rho \\ \rho u \\ \rho v \\ \rho w \\ e \\ s_{x x} \\ s_{y y} \\ s_{z z} \\ s_{x y} \\ s_{x z} \\ s_{y z}\end{array}\right], \mathbf{f}=\left[\begin{array}{c}\rho u \\ \rho u^{2}+p-s_{x x} \\ \rho u v-s_{x y} \\ \rho u w-s_{x z} \\ \left(e+p-s_{x x x}\right) u-s_{x y} v-s_{x z} w \\ u\left(s_{x x}-\frac{4}{3} \mu\right) \\ u\left(s_{y y}+\frac{2}{3} \mu\right) \\ u\left(s_{z z}+\frac{2}{3} \mu\right) \\ u s_{x y}-\mu v \\ u s_{x z}-\mu w \\ u s_{y z}\end{array}\right]$

$$
\mathbf{h}=\left[\begin{array}{c}
\rho w \\
\rho u w-s_{x z} \\
\rho v w-s_{y z} \\
\rho w^{2}+p-s_{z z} \\
\left(e+p-s_{z z}\right) w-s_{y z} v-s_{x z} u \\
w\left(s_{x x}+\frac{2}{3} \mu\right) \\
w\left(s_{y y}+\frac{2}{3} \mu\right) \\
w\left(s_{z z}-\frac{4}{3} \mu\right) \\
w s_{x y} \\
w s_{x z}-\mu u \\
w s_{y z}-\mu v
\end{array}\right]
$$

$\mathrm{g}=\left[\begin{array}{c}\rho v \\ \rho u v-s_{x y} \\ \rho v^{2}+p-s_{y y} \\ \rho v w-s_{y z} \\ \left(e+p-s_{y y}\right) v-s_{x y} u-s_{y z} w \\ v\left(s_{x x}+\frac{2}{3} \mu\right) \\ v\left(s_{y y}-\frac{4}{3} \mu\right) \\ v\left(s_{z z}+\frac{2}{3} \mu\right) \\ v s_{x y}-\mu u \\ v s_{x z} \\ v s_{y z}-\mu w\end{array}\right]$

$$
\mathbf{k}=\left[\begin{array}{c}
0 \\
0 \\
0 \\
0 \\
0 \\
s_{x x}\left(\frac{\partial u}{\partial x}+\frac{\partial v}{\partial y}+\frac{\partial w}{\partial z}\right)+s_{x y}\left(\frac{\partial u}{\partial y}-\frac{\partial v}{\partial x}\right)+s_{x z}\left(\frac{\partial u}{\partial z}-\frac{\partial w}{\partial x}\right)-\lambda s_{x x} \\
s_{x y}\left(\frac{\partial v}{\partial x}-\frac{\partial u}{\partial y}\right)+s_{y y}\left(\frac{\partial u}{\partial x}+\frac{\partial v}{\partial y}+\frac{\partial w}{\partial z}\right)+s_{y z}\left(\frac{\partial v}{\partial z}-\frac{\partial w}{\partial y}\right)-\lambda s_{y y} \\
s_{x z}\left(\frac{\partial w}{\partial x}-\frac{\partial u}{\partial z}\right)+s_{y z}\left(\frac{\partial w}{\partial y}-\frac{\partial v}{\partial z}\right)+s_{z z}\left(\frac{\partial u}{\partial x}+\frac{\partial v}{\partial y}+\frac{\partial w}{\partial z}\right)-\lambda s_{z z} \\
s_{x y}\left(\frac{\partial u}{\partial x}+\frac{\partial v}{\partial y}+\frac{\partial w}{\partial z}\right)-\frac{1}{2}\left(s_{x x}-s_{y y}\right)\left(\frac{\partial u}{\partial y}-\frac{\partial w}{\partial x}\right)+\frac{1}{2} s_{x z}\left(\frac{\partial v}{\partial z}-\frac{\partial w}{\partial y}\right)+\frac{1}{2} s_{y z}\left(\frac{\partial u}{\partial z}-\frac{\partial w}{\partial x}\right)-\lambda s_{x y} \\
s_{x z}\left(\frac{\partial u}{\partial x}+\frac{\partial v}{\partial y}+\frac{\partial w}{\partial z}\right)-\frac{1}{2}\left(s_{x x}-s_{z z}\right)\left(\frac{\partial u}{\partial y}+\frac{\partial w}{\partial x}\right)+\frac{1}{2} s_{x y}\left(\frac{\partial w}{\partial y}+\frac{\partial v}{\partial z}\right)+\frac{1}{2} s_{y z}\left(\frac{\partial u}{\partial z}-\frac{\partial w}{\partial x}\right)-\lambda s_{x y} \\
\left.s_{y y}-s_{z z}\right)\left(\frac{\partial u}{\partial y}-\frac{\partial w}{\partial x}\right)+\frac{1}{2} s_{x z}\left(\frac{\partial v}{\partial x}-\frac{\partial u}{\partial y}\right)+\frac{1}{2} s_{x y}\left(\frac{\partial w}{\partial x}-\frac{\partial u}{\partial z}\right)-\lambda s_{y z}
\end{array}\right]
$$


System (2.1) is closed by adding the equation of state (EOS) in the form

$\varepsilon=\varepsilon(p, \rho)$

In relations (2.1) and (2.2): $p$ - pressure, $\rho$ - density, $\mathrm{u}, \mathrm{v}, \mathrm{w}$ - velocity components with respect to $\mathrm{x}, \mathrm{y}$ and $\mathrm{z} ; \varepsilon-$ is the internal energy per unit mass, $\mathrm{e}-$ is the total mechanical energy per unit volume of the continuous medium, $\mathrm{s}_{x x}, \mathrm{~s}_{y y}, \mathrm{~s}_{z z}, \mathrm{~s}_{x y}, \mathrm{~s}_{x z}, \mathrm{~s}_{y z}$ - are the components of the true Euler deviatoric stress tensor, $\mu-$ is the shear modulus

The first five equations of system (2.1) are the laws of conservation of mass, momentum, and energy. The next six equations are the physical relations of elasticity and plasticity taking into account the rotation of the stress tensor in Euler coordinates (the Jaumann derivative), written in differential form.

To the system (2.1), (2.2), consisting of 11 differential equations for 11 unknown functions $\rho, \mathrm{u}, \mathrm{v}, \mathrm{w}, \varepsilon, \mathrm{s}_{x x}, \mathrm{~s}_{y y}, \mathrm{~s}_{z z}, \mathrm{~s}_{x y}, \mathrm{~s}_{x z}$, $\mathrm{s}_{y z}$, depending on $t, x, y, z$, initial and boundary conditions are added, which are divided into kinematic, dynamic and mixed [10], defined in the general case on various sections of the external boundary of the domain under analysis.

The von Mises flow condition is used as a criterion for the transition from an elastic to a plastic state, according to which, if the inequality

$$
J_{2}=\frac{1}{2}\left(s_{i j} s_{i j}\right) \geq \frac{1}{3} \sigma_{s}{ }^{2}, \quad i, j=x, y, z
$$

where $J_{2}$ is the second invariant of the stress deviator, $\sigma_{\mathrm{s}}-$ is the yield strength of the material under uniaxial tension-compression, then the components of the stress deviator are corrected by "projecting" them onto the yield surface by multiplying them by a factor то $1 / \sqrt{ } \lambda$. The parameter $\lambda$ characterizes the total work of plastic deformations and is calculated by the formula

$$
\lambda=\frac{3}{\sigma_{s}^{2}} J_{2} .
$$

For barotropic processes of medium motion, instead of EOS in the form (2.2), EOS of the $p=p(\rho)$

is used and the energy conservation equation is excluded from (2.1).

The integral form of system (2.1), on the basis of which the difference scheme is constructed, has the form

$$
\oiiint_{\omega} \mathbf{u} d x d y d z+\mathbf{f} d y d z d t+\mathbf{g} d x d z d t+\mathbf{h} d x d y d t=\iiint \int_{\Omega} \mathbf{k} d x d y d z d t
$$

where $\Omega$ - is any closed volume whose surface $\omega$ is homeomorphic to a sphere in four-dimensional space $(\mathrm{x}, \mathrm{y}, \mathrm{z}, \mathrm{t})$.

\section{Finite-dlifference scheme}

The difference relations for system (2.5) are constructed similarly to [1], [2]. The numerical solution area is covered by a regular fixed grid consisting of rectangular parallelepipeds with sides $\mathrm{h}_{x}, \mathrm{~h}_{y}, \mathrm{~h}_{z}$.

Integral relations (2.5) are applied to a space-time cell (STC) in four-dimensional space, a time step is designated $\Delta \mathrm{t}$, space steps $\Delta \mathrm{x}, \Delta \mathrm{y}, \Delta \mathrm{z}$. The subscripts in difference formulas refer to the time instant $t^{\mathrm{n}}$, and the superscripts to the time instant $\mathrm{t}^{(\mathrm{n}+1)}=\mathrm{t}^{\mathrm{n}}+\Delta \mathrm{t}$, integer indices refer to the parameters in the centers of the cells, half-integer ones refer to the centers of the corresponding faces. The following assumptions are assumed to be fulfilled.

On the grounds $\mathrm{t}=\mathrm{t}^{\mathrm{n}}$ and $\mathrm{t}=\mathrm{t}^{\mathrm{n}+1}$ the STC parameters of the medium are constant and equal to some average values at time instants $\mathrm{t}^{\mathrm{n}}$ and $\mathrm{t}^{\mathrm{n}+1}$ accordingly,

$$
\begin{aligned}
& {\left[\left(\rho, p, u, v, w, \varepsilon, s_{x x}, s_{y y}, s_{z z}, s_{x y}, s_{x z}, s_{y z}\right)_{i, j, k}\right],} \\
& {\left[\left(\rho, p, u, v, w, \varepsilon, s_{x x}, s_{y y}, s_{z z}, s_{x y}, s_{x z}, s_{y z}{ }^{i, j, k}\right] .\right.}
\end{aligned}
$$

Pressure $\mathrm{p}$ is determined from the equation of state (2.2).

On the lateral faces of the STC during each interval $\Delta t$ these values are also constant and are denoted by

$\left[\left(\mathrm{R}, \mathrm{P}, \mathrm{U}, \mathrm{V}, \mathrm{W}, \mathrm{E}, \mathrm{S}_{\mathrm{xx}}, \mathrm{S}_{\mathrm{yy}}, \mathrm{S}_{z z}, \mathrm{~S}_{\mathrm{xyy}}, \mathrm{S}_{\mathrm{xz}}, \mathrm{S}_{\mathrm{yz}}\right)_{1}\right]$

These are Riemann's solutions or flux calculating parameters, which are determined from the solution of the Riemann problem of an arbitrary discontinuity across the 1 boundary of the $(\mathrm{i}, \mathrm{j}, \mathrm{k})$ cell, and the values $\mathrm{l}=1,2,3,4,5,6$ correspond to the $(\mathrm{i}-1 / 2, \mathrm{j}, \mathrm{k}),(\mathrm{i}+1 / 2, \mathrm{j}, \mathrm{k}),(\mathrm{i}, \mathrm{j}-1 / 2, \mathrm{k}),(\mathrm{i}, \mathrm{j}+1 / 2, \mathrm{k}),(\mathrm{i}, \mathrm{j}, \mathrm{k}-1 / 2)$, $(\mathrm{i}, \mathrm{j}, \mathrm{k}+1 / 2)$ cell boundaries and are used to determine the corresponding fluxes. Calculation of the fluxes is performed using the one-dimensional Riemann solver of an arbitrary discontinuity. If the 1-th cell boundary belongs to the boundary of the domain of numerical solution, then the 1-th fluxes in this case are calculated using boundary conditions.

After introducing into consideration vectors $\mathbf{U}, \mathbf{F}, \mathbf{G}, \mathbf{H}$, which are a complete analogue of vectors $\mathbf{u}, \mathbf{f}, \mathbf{g}, \mathbf{h}$, but consisting of flow parameters (3.1), the explicit finite-difference equations connecting the unknown at the moment $\mathrm{t}^{\mathrm{n}+1}$ components of the vector $\mathrm{u}$ with the components known at the moment $\mathrm{t}^{\mathrm{n}}$ component $u$ in the cell $\mathrm{i}-1 / 2, \mathrm{j}-1 / 2, \mathrm{k}-1 / 2$, have the following form:

$$
\begin{aligned}
\mathbf{u}_{i, j, k}^{i, j}= & \mathbf{u}_{i, j, k}^{\square}-\frac{\Delta t}{\Delta x}\left(\mathbf{F}_{i+1 / 2, j, k}^{\square}-\mathbf{F}_{i-1 / 2, j, k}^{\square}\right)-\frac{\Delta t}{\Delta y}\left(\mathbf{G}_{i, j+1 / 2, k}^{\square}-\mathbf{G}_{i, j-1 / 2, k}^{\square}\right)- \\
& -\frac{\Delta t}{\Delta z}\left(\mathbf{H}_{i, j, k+1 / 2}^{\square}-\mathbf{H}_{i, j, k-1 / 2}^{\square}\right)+\Delta t\left((1-\alpha) \mathbf{k}_{i, j, k}^{\square}+\alpha \mathbf{k}_{[., j}^{i, j}\right)
\end{aligned}
$$

The solution of the linearized Riemann problem taking into account shear stresses

The solution of the Riemann problem is carried out on the basis of a simplified system of equations in the elastic approximation obtained from (2.1), if assume $\lambda=0$.

We linearize the initial system $(2.1,2.2)$ in the vicinity of a certain $\mathrm{U}=\mathrm{U}_{0}$ and assume that the motion occurs along one of the axes and all unknown functions depend on only one spatial variable, for example, $x$. We get a system of eleven equations

$$
\partial \mathbf{U} / \partial t+\mathbf{A} \partial \mathbf{U} / \partial x=0
$$

where

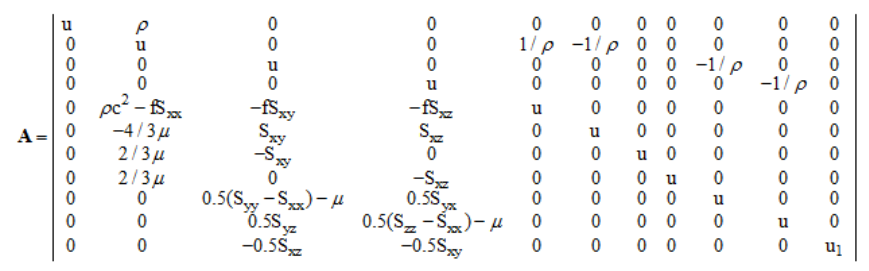


Here $\mathrm{c}^{2}=\left(\partial \mathrm{p} / \partial \rho_{\mathrm{s}}, \mathrm{f}=\frac{1}{\rho(\partial \varepsilon / \partial \mathrm{p})_{\rho}}\right.$ - are the result of

linearization of the EOS (2.2).

The Riemann problem of an arbitrary discontinuity is the Cauchy problem for system (4.1) with piecewise constant initial conditions $\mathbf{U}=\mathbf{U}_{(1)}, x<x_{0}, \mathbf{U}=\mathbf{U}_{(2)}, \mathrm{x}>\mathrm{x}_{0}$.

According to the general theory of an arbitrary discontinuity [24], for $\mathrm{t}>0$ the discontinuity disintegrates forming one of the possible stable configurations of discontinuities separating the regions of continuous flow. The solution to the system of linear differential equations (4.1) is sought under the assumption of its self-similarity. After the introduction of a self-similar variable $\xi=\mathrm{x} / \mathrm{t}$ system (4.1) is transformed to a system of ordinary differential equations

$(\mathbf{A}-\boldsymbol{\xi} \mathbf{E}) \mathrm{dU} / \mathrm{dt}=0$, where $\mathrm{E}-$ is the identity matrix.

Nontrivial solutions to this system are:

$\xi_{1}=\mathrm{u}+\mathrm{a}, \quad \xi_{2}=\mathrm{u}-\mathrm{a}, \quad \xi_{3}=\mathrm{u}+\beta_{\mathrm{y}}, \quad \xi_{4}=\mathrm{u}-\beta_{\mathrm{y}}, \quad \xi_{5}=\mathrm{u}+\beta_{\mathrm{z}}$, $\xi 6=\mathrm{u}-\beta_{\mathrm{z}}, \quad \xi_{\mathrm{7}}=\mathrm{u}, \xi_{8}=\mathrm{u}, \quad \xi_{\mathrm{g}}=\mathrm{u}, \quad \xi_{10}=\mathrm{u}, \quad \xi_{11}=\mathrm{u}$.

They are the velocities of weak discontinuities forming a stable configuration. Here

$\mathrm{a}^{2}=\mathrm{c}^{2}+\frac{4 / 3 \mu-\mathrm{fS}_{\mathrm{xx}}}{\rho}, \beta_{\mathrm{y}}=\sqrt{\frac{\mu+3 / 4 \mathrm{~S}_{\mathrm{xx}}}{\rho}-0.5 \sqrt{\frac{0.25^{*}\left(\mathrm{~S}_{\mathrm{yy}}-\mathrm{S}_{\mathrm{z}}\right)^{2}+\mathrm{S}_{\mathrm{yz}}^{2}}{\rho^{2}}}}$

$\beta_{z}=\sqrt{\frac{\mu+3 / 4 \mathrm{~S}_{\mathrm{xx}}}{\rho}+0.5 \sqrt{\frac{0.25^{*}\left(\mathrm{~S}_{\mathrm{yy}}-\mathrm{S}_{\mathrm{zz}}\right)^{2}+\mathrm{S}_{\mathrm{yz}}{ }^{2}}{\rho^{2}}}}$

In the plane of $(\mathrm{x}, \mathrm{t})$ the trajectory of discontinuities (characteristics) are represented by rays emanating from the point $\mathrm{x}=\mathrm{x}_{0}$, and divide the half-plane $\mathrm{t}>0$ into 8 zones (see figure 1).

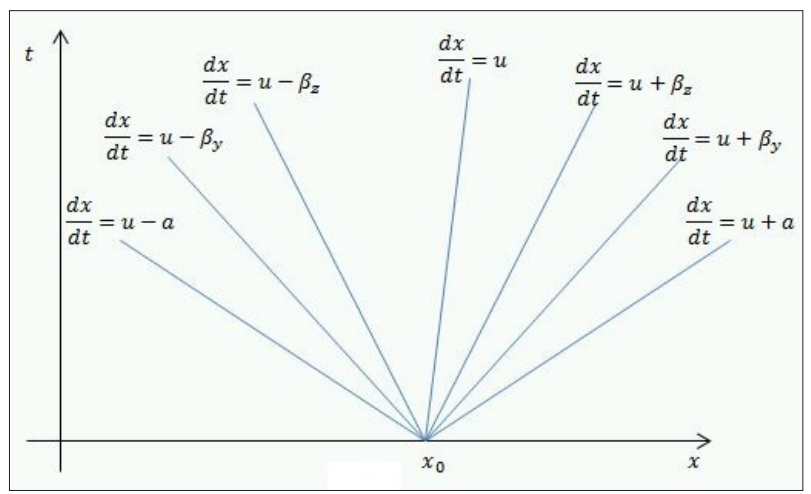

Figure 1

The relationships on these characteristics $\mathrm{R}_{l}, l=\overline{1,11}$ are as follows:

$$
\begin{aligned}
& \mathrm{R}_{\mathrm{l}}=[\rho \mathrm{a}] \mathrm{u}-\left[\mathrm{a}(1+\mathrm{f}) \frac{\mathrm{S}_{\mathrm{xy}}\left(\mathrm{a}^{2}-\mathrm{b}_{3}{ }^{2}\right)-\mathrm{S}_{\mathrm{xz}}{ }^{*} 0.5 \mathrm{~S}_{\mathrm{yz}} / \rho}{\left(\mathrm{a}^{2}-\mathrm{b}_{2}{ }^{2}\right)\left(\mathrm{a}^{2}-\mathrm{b}_{3}{ }^{2}\right)-0.25 \mathrm{~S}_{\mathrm{yz}}{ }^{2} / \rho^{2}}\right] \\
& \mathrm{v}-\left[\mathrm{a}(1+\mathrm{f}) \frac{\mathrm{S}_{\mathrm{xz}}\left(\mathrm{a}^{2}-\mathrm{b}_{2}{ }^{2}\right)-\mathrm{S}_{\mathrm{xy}}{ }^{*} 0.5 \mathrm{~S}_{\mathrm{yz}} / \rho}{\left(\mathrm{a}^{2}-\mathrm{b}_{2}{ }^{2}\right)\left(\mathrm{a}^{2}-\mathrm{b}_{3}{ }^{2}\right)-0.25 \mathrm{~S}_{\mathrm{yz}}{ }^{2} / \rho^{2}}\right] \mathrm{w}+
\end{aligned}
$$

$+\mathrm{p}-\mathrm{S}_{\mathrm{xx}}+\left[(1+\mathrm{f}) \frac{\rho \mathrm{S}_{\mathrm{xy}}\left(\mathrm{a}^{2}-\mathrm{b}_{3}{ }^{2}\right)-\mathrm{S}_{\mathrm{xz}} * 0.5 \mathrm{~S}_{\mathrm{yz}}}{\rho^{2}\left(\mathrm{a}^{2}-\mathrm{b}_{2}{ }^{2}\right)\left(\mathrm{a}^{2}-\mathrm{b}_{3}{ }^{2}\right)-0.25 \mathrm{~S}_{\mathrm{yz}}{ }^{2}}\right] \mathrm{S}_{\mathrm{xy}}$
$+\left[(1+\mathrm{f}) \frac{\rho \mathrm{S}_{\mathrm{xz}}\left(\mathrm{a}^{2}-\mathrm{b}_{2}{ }^{2}\right)-\mathrm{S}_{\mathrm{xy}} * 0.5 \mathrm{~S}_{\mathrm{yz}}}{\rho^{2}\left(\mathrm{a}^{2}-\mathrm{b}_{2}{ }^{2}\right)\left(\mathrm{a}^{2}-\mathrm{b}_{3}{ }^{2}\right)-0.25 \mathrm{~S}_{\mathrm{yz}}{ }^{2}}\right] \mathrm{S}_{\mathrm{xz}}$
$\mathrm{b}_{2}{ }^{2}=\frac{\mu+0.5\left(\mathrm{~S}_{\mathrm{xx}}-\mathrm{S}_{\mathrm{yy}}\right)}{\rho} ; \mathrm{b}_{3}{ }^{2}=\frac{\mu+0.5\left(\mathrm{~S}_{\mathrm{xx}}-\mathrm{S}_{\mathrm{zz}}\right)}{\rho}$

$\mathrm{R}_{2}=[-\rho \mathrm{a}] \mathrm{u}+\left[\rho \mathrm{a}(1+\mathrm{f}) \frac{\rho \mathrm{S}_{\mathrm{xy}}\left(\mathrm{a}^{2}-\mathrm{b}_{3}{ }^{2}\right)-\mathrm{S}_{\mathrm{xz}}{ }^{*} 0.5 \mathrm{~S}_{\mathrm{yz}}}{\rho^{2}\left(\mathrm{a}^{2}-\mathrm{b}_{2}{ }^{2}\right)\left(\mathrm{a}^{2}-\mathrm{b}_{3}{ }^{2}\right)-0.25 \mathrm{~S}_{\mathrm{yz}}{ }^{2}}\right]$

$\mathrm{v}+\left[\rho \mathrm{a}(1+\mathrm{f}) \frac{\rho \mathrm{S}_{\mathrm{xz}}\left(\mathrm{a}^{2}-\mathrm{b}_{2}^{2}\right)-\mathrm{S}_{\mathrm{xy}} * 0.5 \mathrm{~S}_{\mathrm{yz}}}{\rho^{2}\left(\mathrm{a}^{2}-\mathrm{b}_{2}^{2}\right)\left(\mathrm{a}^{2}-\mathrm{b}_{3}^{2}\right)-0.25 \mathrm{~S}_{\mathrm{yz}}{ }^{2}}\right] \mathrm{w}+$

$+\mathrm{p}-\mathrm{S}_{\mathrm{xx}}+\left[(1+\mathrm{f}) \frac{\rho \mathrm{S}_{\mathrm{xy}}\left(\mathrm{a}^{2}-\mathrm{b}_{3}{ }^{2}\right)-\mathrm{S}_{\mathrm{xz}} * 0.5 \mathrm{~S}_{\mathrm{yz}}}{\rho^{2}\left(\mathrm{a}^{2}-\mathrm{b}_{2}{ }^{2}\right)\left(\mathrm{a}^{2}-\mathrm{b}_{3}{ }^{2}\right)-0.25 \mathrm{~S}_{\mathrm{yz}}{ }^{2}}\right] \mathrm{S}_{\mathrm{xy}}$

$+\left[(1+\mathrm{f}) \frac{\rho \mathrm{S}_{\mathrm{xz}}\left(\mathrm{a}^{2}-\mathrm{b}_{2}{ }^{2}\right)-\mathrm{S}_{\mathrm{xy}} * 0.5 \mathrm{~S}_{\mathrm{yz}}}{\rho^{2}\left(\mathrm{a}^{2}-\mathrm{b}_{2}{ }^{2}\right)\left(\mathrm{a}^{2}-\mathrm{b}_{3}{ }^{2}\right)-0.25 \mathrm{~S}_{\mathrm{yz}}{ }^{2}}\right] \mathrm{S}_{\mathrm{xz}}$

$\mathrm{R}_{3}=\left[\beta_{\mathrm{y}} \rho\right] \mathrm{v}-\left[\beta_{\mathrm{y}} \rho \mathrm{C}\right] \mathrm{w}-\mathrm{S}_{\mathrm{xy}}+[\mathrm{C}] \mathrm{S}_{\mathrm{xz}}$

$\mathrm{R}_{4}=-\left[\beta_{\mathrm{y}} \rho\right] \mathrm{v}+\left[\beta_{\mathrm{y}} \rho \mathrm{C}\right] \mathrm{w}-\mathrm{S}_{\mathrm{xy}}+[\mathrm{C}] \mathrm{S}_{\mathrm{xz}}$

$\mathrm{R}_{5}=\left[\beta_{\mathrm{z}} \rho \mathrm{C}\right] \mathrm{v}-\left[\beta_{\mathrm{z}} \rho\right] \mathrm{w}-[\mathrm{C}] \mathrm{S}_{\mathrm{xy}}+\mathrm{S}_{\mathrm{xz}}, \quad \mathrm{C}=\sqrt{1-\frac{2}{1+\sqrt{1+\frac{4 \mathrm{~S}_{\mathrm{yz}}{ }^{2}}{\left(\mathrm{~S}_{\mathrm{yy}}-\mathrm{S}_{\mathrm{zz}}\right)^{2}}}}}$

$\mathrm{R}_{6}=-\left[\beta_{\mathrm{z}} \rho \mathrm{C}\right] \mathrm{v}+\left[\beta_{\mathrm{z}} \rho\right] \mathrm{w}-\mathrm{CS}_{\mathrm{xy}}+\mathrm{S}_{\mathrm{xz}}$

$\mathrm{R}_{7}=\left[\mathrm{a}^{2}\right] \rho-\mathrm{p}+\mathrm{S}_{\mathrm{xx}}+\left[\frac{(1+\mathrm{f})\left(\mathrm{S}_{\mathrm{xy}} \rho \mathrm{b}_{3}{ }^{2}+0.5 \mathrm{~S}_{\mathrm{yz}} \mathrm{S}_{\mathrm{xz}}\right)}{\rho \mathrm{b}_{2}{ }^{2} \rho \mathrm{b}_{3}{ }^{2}-0.25 \mathrm{~S}_{\mathrm{yx}}{ }^{2}}\right] \mathrm{S}_{\mathrm{xy}}$

$+\left[\frac{(1+\mathrm{f})\left(\rho \mathrm{b}_{2}{ }^{2} \mathrm{~S}_{\mathrm{xz}}+0.5 \mathrm{~S}_{\mathrm{yz}} \mathrm{S}_{\mathrm{xy}}\right)}{\rho \mathrm{b}_{2}{ }^{2} \rho \mathrm{b}_{3}^{2}-0.25 \mathrm{~S}_{\mathrm{yz}}^{2}}\right] \mathrm{S}_{\mathrm{xz}}$

$\mathrm{R}_{8}=\left[4 / 3 \frac{\mu}{\rho}\right] \rho+\mathrm{S}_{\mathrm{xx}}+\left[\frac{\mathrm{S}_{\mathrm{xy}} \rho \mathrm{b}_{3}{ }^{2}+0.5 \mathrm{~S}_{\mathrm{yz}} \mathrm{S}_{\mathrm{xz}}}{\rho \mathrm{b}_{2}{ }^{2} \rho \mathrm{b}_{3}{ }^{2}-0.25 \mathrm{~S}_{\mathrm{yz}}{ }^{2}}\right] \mathrm{S}_{\mathrm{xy}}$

$+\left[\frac{\rho \mathrm{b}_{2}{ }^{2} \mathrm{~S}_{\mathrm{xz}}+0.5 \mathrm{~S}_{\mathrm{yz}} \mathrm{S}_{\mathrm{xy}}}{\rho \mathrm{b}_{2}^{2} \rho \mathrm{b}_{3}{ }^{2}-0.25 \mathrm{~S}_{\mathrm{yz}}{ }^{2}}\right] \mathrm{S}_{\mathrm{xz}}$

$\mathrm{R}_{9}=\left[-2 / 3 \frac{\mu}{\rho}\right] \rho+\mathrm{S}_{\mathrm{yy}}+\left[\frac{-\mathrm{S}_{\mathrm{xy}} \rho \mathrm{b}_{3}{ }^{2}}{\rho \mathrm{b}_{2}{ }^{2} \rho \mathrm{b}_{3}{ }^{2}-0.25 \mathrm{~S}_{\mathrm{yz}}{ }^{2}}\right] \mathrm{S}_{\mathrm{xy}}$

$+\left[\frac{-0.5 \mathrm{~S}_{\mathrm{yz}} \mathrm{S}_{\mathrm{xy}}}{\rho \mathrm{b}_{2}^{2} \rho \mathrm{b}_{3}^{2}-0.25 \mathrm{~S}_{\mathrm{yz}}^{2}}\right] \mathrm{S}_{\mathrm{xz}}$ 


$$
\begin{aligned}
& \mathrm{R}_{10}=\left[-2 / 3 \frac{\mu}{\rho}\right] \rho+\mathrm{S}_{\mathrm{zz}}+\left[\frac{-0.5 \mathrm{~S}_{\mathrm{yz}} \mathrm{S}_{\mathrm{xz}}}{\rho \mathrm{b}_{2}{ }^{2} \rho \mathrm{b}_{3}{ }^{2}-0.25 \mathrm{~S}_{\mathrm{yz}}{ }^{2}}\right] \mathrm{S}_{\mathrm{xy}} \\
& +\left[\frac{-\rho \mathrm{b}_{2}{ }^{2} \mathrm{~S}_{\mathrm{xz}}}{\rho \mathrm{b}_{2}{ }^{2} \rho \mathrm{b}_{3}{ }^{2}-0.25 \mathrm{~S}_{\mathrm{yz}}{ }^{2}}\right] \mathrm{S}_{\mathrm{xz}} \\
& \mathrm{R}_{11}=\left[\frac{-0.5 \mathrm{~S}_{\mathrm{xz}} \rho \mathrm{b}_{3}{ }^{2}-0.25 \mathrm{~S}_{\mathrm{yz}} \mathrm{S}_{\mathrm{xy}}}{\rho \mathrm{b}_{2}{ }^{2} \rho \mathrm{b}_{3}{ }^{2}-0.25 \mathrm{~S}_{\mathrm{yz}}{ }^{2}}\right] \mathrm{S}_{\mathrm{xy}} \\
& +\left[\frac{-0.5 \rho \mathrm{b}_{2}{ }^{2} \mathrm{~S}_{\mathrm{xy}}-0.25 \mathrm{~S}_{\mathrm{yz}} \mathrm{S}_{\mathrm{xz}}}{\rho \mathrm{b}_{2}{ }^{2} \rho \mathrm{b}_{3}{ }^{2}-0.25 \mathrm{~S}_{\mathrm{yz}}{ }^{2}}\right] \mathrm{S}_{\mathrm{xz}}+\mathrm{S}_{\mathrm{yz}}
\end{aligned}
$$

Here the values in square brackets are the linearization coefficients, determined by the averaged parameters in the cells. The stability condition for the constructed first-order accuracy scheme is the inequality

$$
\frac{|u|+a}{\Delta x}+\frac{|v|+a}{\Delta y}+\frac{|w|+a}{\Delta z} \leq \frac{1}{\Delta t} .
$$

This condition is classical for difference schemes with the splitting of operators in directions $[2,24]$.

\section{High order accuracy scheme}

The second-order accuracy scheme is constructed in the same way as for the equations of gas dynamics [16, 17]. For this, before solving the Riemann problem, it is necessary to additionally assume a linear distribution of all functions between the centers of difference cells. In contrast to the equations of gas dynamics, in this case, the Riemann invariants are interpolated from the center of the cells $[18,19]$. The coordinates of the interpolation points are defined as the boundaries of the regions of influence of the corresponding invariants on the position of the interface at the time $\Delta t / 2$ as follows (see Fig. 2):

$$
x_{n}=\frac{\left(x_{i-1}+x_{i}\right)}{2}-\left(c_{n}-w\right) \frac{\Delta t}{2}, n=1, \ldots, 11 \text {, }
$$

where $w$ is the speed of the face and

$$
\begin{aligned}
& c_{1}=u+a ; c_{2}=u-a ; c_{3}=u+\beta_{y} ; c_{4}=u-\beta_{y} ; \\
& c_{5}=u+\beta_{z} ; c_{6}=u-\beta_{z} ; c_{7}=c_{8}=c_{9}=c_{10}=c_{11}=u ;
\end{aligned}
$$

Denote the interpolated invariants by the superscripts ' $m$ ', respectively

$$
R_{n}^{m}=R_{n}^{i-1}+\frac{R_{n}^{i}-R_{n}^{i-1}}{x_{i}-x_{i-1}}\left(x_{n}-x_{i-1}\right), n=1, \ldots, 11
$$

The obtained values of the invariants are used to determine the primitive parameters and fluxes. The stage of numerical integration of equations (stage corrector) remains unchanged.

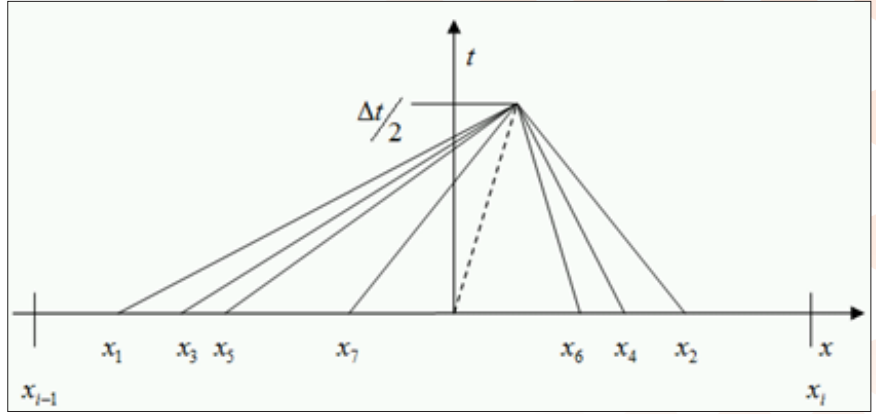

Figure 2

The following notations are introduced:

$$
\begin{aligned}
& x_{1}=0.5^{*} \Delta t^{*}(w-u-a) ; x_{3}=0.5^{*} \Delta t^{*}\left(w-u-\beta_{y}\right) ; x_{5}=0.5^{*} \Delta t^{*}\left(w-u-\beta_{z}\right) ; \\
& x_{2}=0.5^{*} \Delta t^{*}(w-u+a) ; x_{4}=0.5^{*} \Delta t^{*}\left(w-u+\beta_{y}\right) ; x_{6}=0.5^{*} \Delta t^{*}\left(w-u+\beta_{z}\right) ; \\
& x_{7}=x_{8}=x_{9}=x_{10}=x_{11}=0.5^{*} \Delta t^{*}(w-u) ;
\end{aligned}
$$

The same solution is used to implement the basic boundary conditions of a "rigid wall" (symmetric parameters are set with the opposite normal speed), "fixed boundary" (3 velocity components are set at the boundary), "P-boundary" (normal stresses and zero shear stresses are set) and "P-boundary" with friction (normal stresses and tangential stresses associated with the Coulomb law with normal are specified). Free borders are a special case of "P-boundaries".

As shown in [25], using the solution of Riemann problem in the elastic approximation with a second order of accuracy at the "predictor" step of the difference scheme and taking into account plastic deformation at the "corrector" step in accordance with the principle of splitting into physical processes preserves the second order of accuracy as a whole.

3D multi mesh algorithm for calculating problems with curved boundary surfaces.

3D multi mesh algorithm for calculating the contact interaction of media and construction in Euler variables was proposed in [26] and consists of a sequence of the following steps.

1. Calculation domains (structures and environments) are set in the form of surfaces from sets of triangles with the necessary accuracy - in the form of STL files containing the coordinates of external normals and vertices of triangles. These files can be obtained by CAD - tools, for example, such as COMPASS or SolidWork.

2. Each computational domain with curvilinear boundaries is enclosed in a bordering rectangular parallelepiped with surfaces parallel to the coordinate planes and is covered by a regular Cartesian grid. Only the sizes of the Cartesian grid cells are specified. We get four types of cells for the computational domain, the first type is cells intersecting by surface triangles or boundary cells, the second type is cells outside the surface, the third type is cells inside the surface, for integrating which with a given approximation, a difference stencil of whole cells is enough (in our case stencil $3 \times 3 \times 3$ ), the fourth type - cells, for the integration of which there is not enough difference stencil from whole cells inside the surface. The parameters in the cells of the third kind are determined on a new time layer in a fixed 
Cartesian mesh according to [19].

3. On each triangle of the surface, a local Cartesian mesh $3 \times 3 \times 3$ is built inward of the volume from this surface, with planes parallel to the plane of the corresponding triangle. The cell sizes of this local 3D mesh are taken close to Cartesian. In the case of contact of a triangle with another subdomain (proximity to any of its triangles), the local grid symmetrically extends outward from the plane of the triangle by another $3 \times 3 \times 3$ cells. This $3 \times 3 \times 6$ stencil is enough to integrate the four central cells with the second order of accuracy according to the modified Godunov's scheme. The values of the parameters of the local grid are determined by interpolating the parameters from the main grid. The Riemann problem at the contact boundaries of the media is calculated. The results of the Riemann's solver are speeds and forces on a half of time step in the center of the triangle. With normal speed, we move the contact boundary to the new time step, we get a local grid on the new time step. We carry out standard Euler-Lagrangian integration of parameters in moving grids. With this movement of the local grid, the moving volumes necessary for calculating the flows and integration are calculated exactly.

4. Using the velocities in the center of each triangle obtained from the solution of the Riemann problem at stage 3, we calculate with weights proportional to the areas of the velocity triangles at the vertices of the triangles of the STL file and move them to the new time step. We get the position of the surface on the new time step (new STL file).

5. We determine the types of cells inside the new position of the surface and interpolate the parameters from cells of type 3 and cells of the boundary layer into cells of type 4 .

6. Next, we rebuild the basic parallelepiped in accordance with the new STL file (shift by the required number of cells so that the computational domain remains inside the parallelepiped), thus completing the computational step.

The stated numerical technique and algorithm for calculating three-dimensional elastoplastic problems are implemented on the Lobachevsky multiprocessor supercomputer (Lobachevsky NNSU).

Test calculations

Half-plate impact (Wilkins's Problemt)

The problem of a high-speed impact of a plate on an initially stationary halfspace is considered $[27,20]$. The material of the plate and the barrier is aluminum, the thickness of the plate is $5 \mathrm{~mm}$, the left surface of the plate is free, the external pressure is $0.1 \mathrm{MPa}$. For aluminum, the following equation of state is used: $\mathrm{P}(\rho)=72 *\left(\rho / \rho_{0}-1\right)+172 *\left(\rho / \rho_{0}-1\right)^{2}+40 *\left(\rho / \rho_{0}-1\right)^{3}$ where $\mathrm{P}(\rho)$ hydrostatic pressure in GPa, $\rho_{0}=2700 \mathrm{~kg} / \mathrm{m}^{3}$ shear modulus $\mu=24.8 \mathrm{GPa}$, yield strength $\sigma_{\mathrm{S}}=0.2976 \mathrm{GPa}$. The calculations were carried out for collision velocities of 0.8 $\mathrm{km} / \mathrm{s}$ on a uniform difference grid of $500 \times 500 \times 500$ cells (10 cells per millimeter). The thickness of the plate was taken 50 cells. The initial position of the contact boundary $x_{k}=0.5 \mathrm{~cm}$.
Figure 3 shows the distribution of normal stresses in the plate and half-space at various points in time. The solid line shows the solution according to the scheme of Godunov of the second order of accuracy, the dotted line is the first order of accuracy, and the dot-and-dot line is according to the Wilkins scheme, which has the second order of accuracy [27]. A good agreement of the solution according to the second-order accuracy scheme with the Wilkins solution is observed. The solution according to the first-order accuracy scheme does not adequately describe the amplitudes of wave fronts and contact discontinuities.
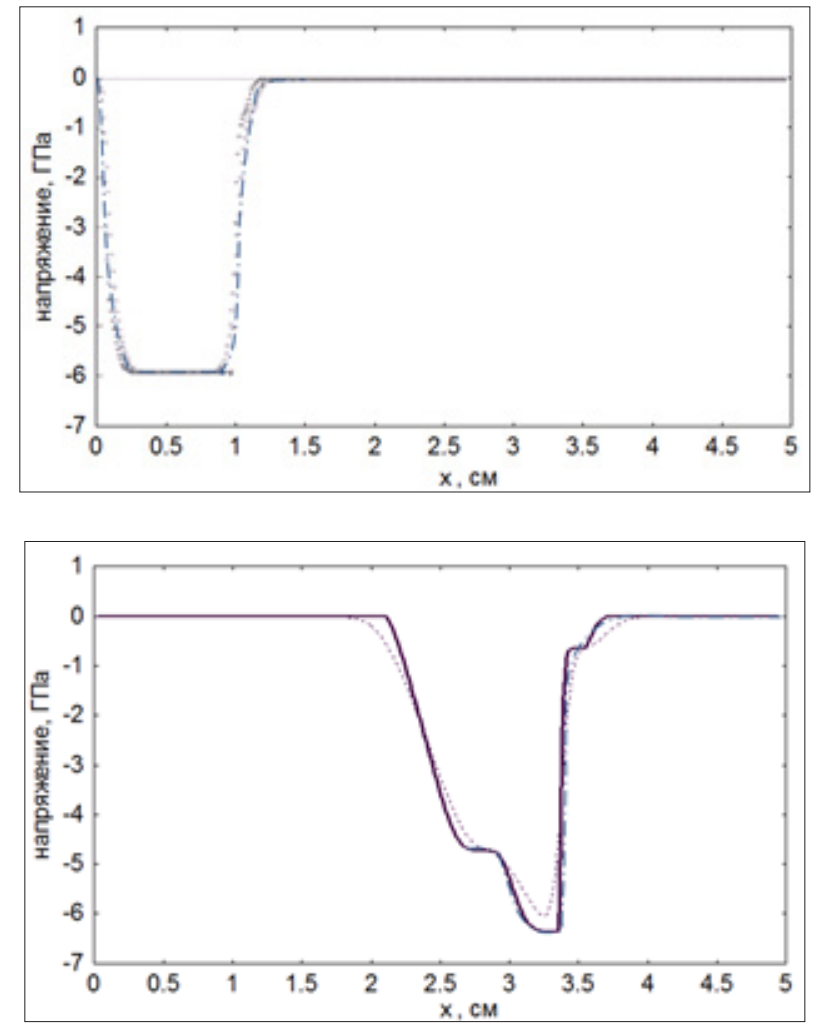

Figure.3

Simulation of forced axisymmetric vibrations of an elastic disk A steel disk with a height of $\mathrm{H}=1.5 \mathrm{~cm}$ and a radius of $\mathrm{R}=$ $14.85 \mathrm{~cm}$ (Fig. 4) is loaded on the lower surface with constant pressure in the direction of the axis Oz. Material properties:, $\rho_{0}=7.8 \mathrm{~g} / \mathrm{cm}^{3}, E=210 \mathrm{GPa}$, Poisson's ratio $v=0.3$. Boundary conditions: rigid fastening along the contour of the plate, pressure $\mathrm{p}=0.17 \mathrm{GPa}$ was set on the lower surface, and the upper surface is a free boundary $p=0$. The cell size is $0.1 \mathrm{~cm}$, the results of calculations by the proposed method were compared with the numerical calculation by LS-DYNA. 


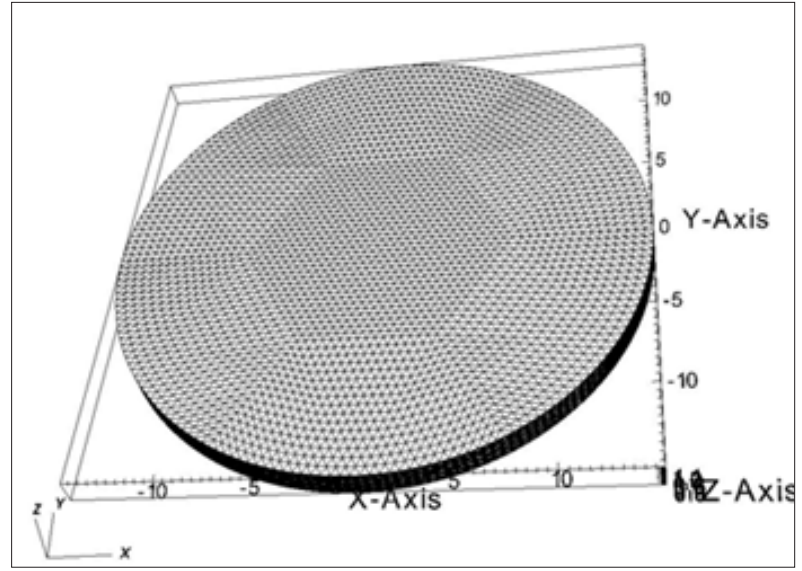

Figure 4: STL file (surface) of the disk

Figure 5 shows the time dependence of the speed of the center of the upper surface of the plate. The numerical solution according to the scheme of Godunov of the second order of accuracy is shown in red, the solution in LS-DYNA is shown in blue. There is a correspondence of decisions on amplitudes and phases of oscillations.

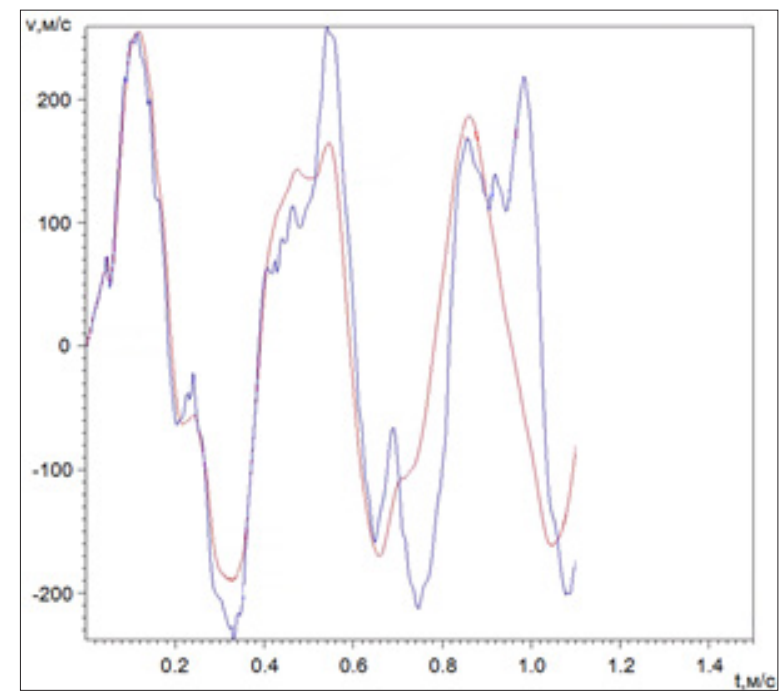

Figure 5

Simulation of the impact and penetration of a copper striker into an aluminum barrier.

A copper cylinder with a height of $\mathrm{H}=2 \mathrm{~cm}$, a radius of $\mathrm{R}=0.4$ $\mathrm{cm}$, an initial vertical speed of $870 \mathrm{~m} / \mathrm{s}$, an aluminum cylinder with a height of $\mathrm{H} 2=6 \mathrm{~cm}$, a radius of $\mathrm{R} 2=3 \mathrm{~cm}$ on a solid base (Fig. 6). Material properties: annealed copper $\rho 0=8.9$ $\mathrm{g} / \mathrm{cm} 3$, bulk modulus $\mathrm{K}=178 \mathrm{GPa}$, shear modulus $\mathrm{G}=48.7$ $\mathrm{GPa}$, yield strength $\sigma_{\mathrm{s}}=75 \mathrm{MPa}$, annealed aluminum $\rho_{0}=2.7 \mathrm{~g} /$ $\mathrm{cm} 3$, bulk modulus $\mathrm{K}=63.9 \mathrm{GPa}$, shear modulus $\mathrm{G}=27 \mathrm{GPa}$, $\sigma_{\mathrm{s}}=80 \mathrm{MPa}$. The ideal ductility of both materials is assumed. The aluminum cylinder stands on a rigid base. The cell size for copper is $0.011 \mathrm{~cm}$, for aluminum $0.025 \mathrm{~cm}$. The results of calculations by the proposed method were compared with the experimental data of Zlatin [28]. Large deformations are observed in both the striker and the target.
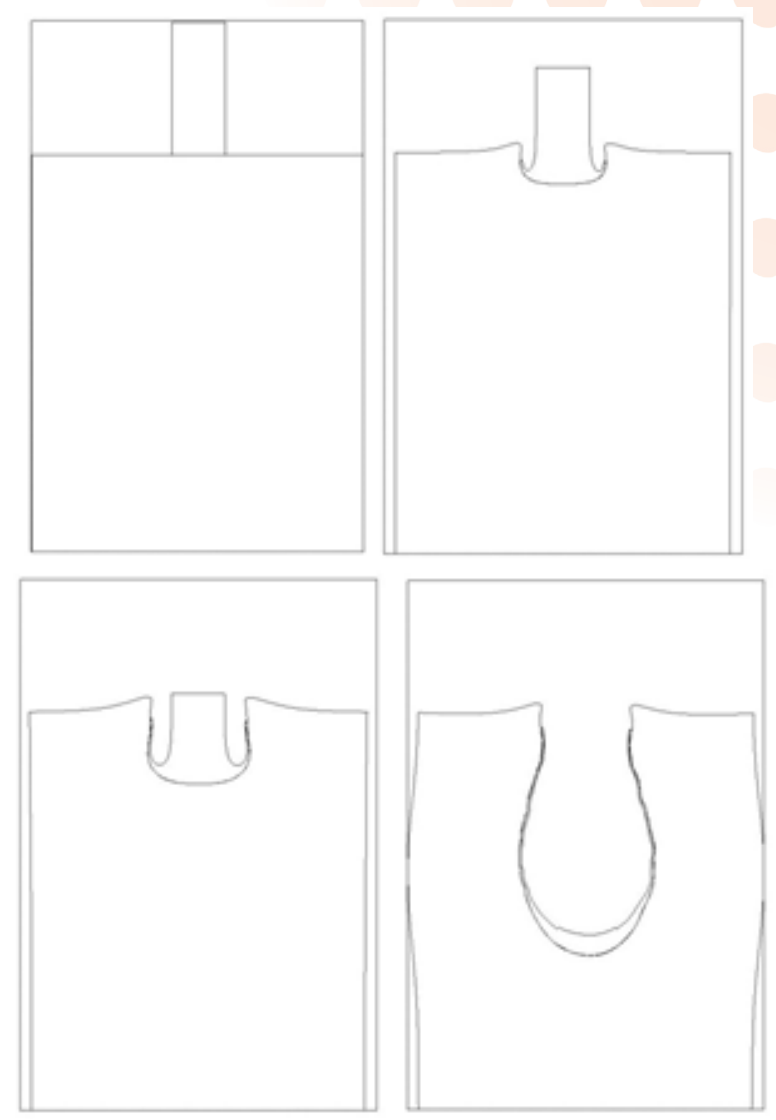

Figure 6

Figure 6 shows the contours of the projectile and the target in axial section at time instants of $0,9,21$, and $100 \mu$ s. The stopping time is $\mathrm{tk}=100 \mu \mathrm{s}$, the final penetration depth - $=3.6$ $\mathrm{cm}$ of the impactor is close to experimental [28]. In fig. 7, in the dimensionless Zlatin coordinates $(\mathrm{t} / \mathrm{tk}, \mathrm{h} / \mathrm{H})$, the results of three-dimensional calculations of the axisymmetric problem are shown in red, the calculations using the Lagrangian-Eulerian method in moving curvilinear grids made by the UPSGOD two-dimensional software complex [29] are blue, the experimental results are shown in green [28].

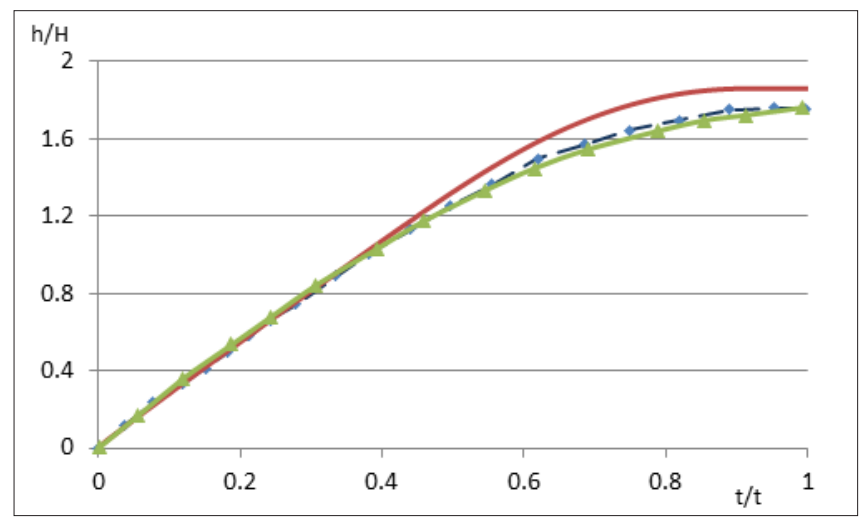

Figure 7

Figure 8 shows the results of the calculation of a three-dimensional non-axisymmetric problem with the same parameters of material of the projectile and the target as in previous calculations upon impact with the side face of a copper cylinder at the 
same vertical speed. The projectile is marked in red, and the target in blue. In contrast to the axisymmetric problem, flattening of the projectile is observed with the formation of a side stream beyond the target, i.e. the process becomes substantially three-dimensional.
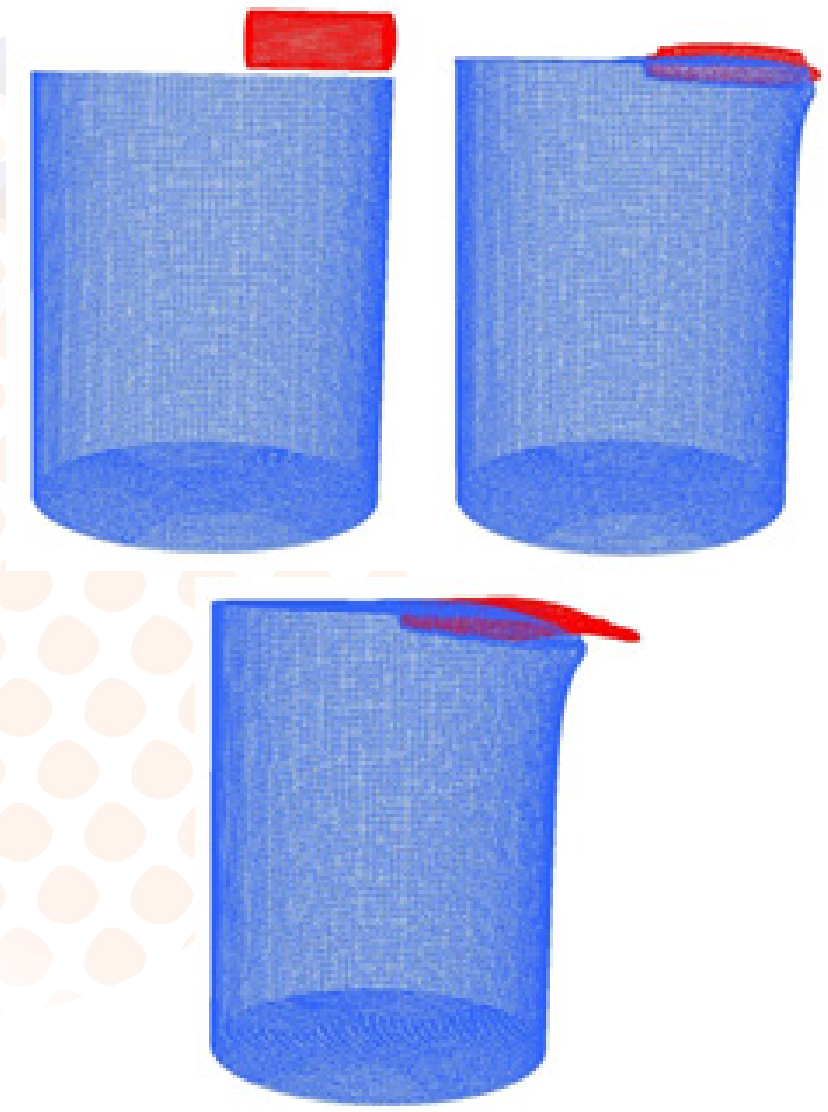

Figure. 8

\section{Conclusion}

A numerical technique for solving three-dimensional wave elastoplastic problems is presented, based on a modified finite-difference scheme of Godunov with increased accuracy and a multigrid calculation algorithm. The numerical technique makes it possible to obtain solutions of three-dimensional nonlinear problems of impact interaction with large deformations and displacements in Euler variables using a multigrid algorithm. Comparison of the obtained solutions with the known solutions by the Eulerian-Lagrangian and Lagrangian methods, as well as with experimental data, shows the efficiency and sufficient accuracy of the presented three-dimensional methodology.

\section{References}

1. Godunov S.K., Zabrodin A.V., Prokopov G.P. (1961) The difference scheme for two-dimensional unsteady problems of gas dynamics and the calculation of the flow around a departing shock wave // Journal of Computational Mathematics and Mathematical Physics, 1961, 1(6): 1020-1050.

2. Godunov S.K., Zabrodin A.V., Ivanov M.Ya. et al. (1976) Numerical solution of multidimensional problems of gas dynamics. M .: Nauka, 1976.

3. S.L. Gavrilyuk, N. Favrie, R. Saurel (20080 Modeling wave dynamics of compressible elastic materials, J. Com- put. Phys. 227 (5): 2941-2969.

4. Cheban V. G., Russu I. V (1976) Numerical methods for solving problems of the dynamic theory of elasticity. Chisinau: Stinnitsa, 1976.

5. P.T. Barton, D. Drikakis, E. Romenski, V.A. Titare (2009) Exact and approximate solutions of Riemann problems in non-linear elasticity, J. Comput. Phys. 228(18): 70467068 .

6. Abuzyarov M.Kh., Bazhenov V.G., Kotov V.L., Kochetkov A.V., Krylov S.V., Feldgun V.R (2000) The method of disintegration of discontinuities in the dynamics of elastoplastic media // Journal of Computational Mathematics and Mathematical Physics, 2000, 40(6): 940-953.

7. I.S. Menshov, A.V. Mishchenko, A.A. Serezhkin (2013) Numerical simulation of elastoplastic flows by the Godunov method on moving Euler networks // Mat. Modeling, 25 (2013), 89-108.

8. L. Michael, N. Nikiforakis. (2016) A hybrid formulation for the numerical simulation of condensed phase explosives, Journal of Computational Physics 316 (2016): 193-217.

9. M.Kh. Abuzyarov, E.G. Glazova, A.V. Kochetkov, S.V. Krylov, V.I. Romanov, M.A. Syrunin (2010) Modeling the interaction of shock waves with deformable gas-tight barriers / // Problems of strength and ductility: Interuniversity. Sat / Nizhny Novgorod. un-t 2010. Issue. 72.S. 120-129.

10. L. Michael, N. Nikiforakis. (2018) A multi-physics methodology for the simulation of the two-way interaction of reactive flow and elastoplastic structural response, $J$. Comput. Phys. 367: 1-27.

11. Kopchenov V.I., Kraiko A.N. (1983) A second-order monotone difference scheme for hyperbolic systems with two independent variables // Zh. calculation mate. and mat. physical 1983. T. 23(4): 848 - 859.

12. Rodionov A.V (1987) Improving the approximation order of K. Godunov's scheme // Zh. calculation mate. and mat. physical 1987.27(12): 1853 - 1860.

13. Moiseev N.Ya. (1988) About one way to increase the accuracy of solutions in difference schemes constructed on the basis of the method of K. Godunov // VANT, series: Methods and programs num. problem solving mat. Physics 1988, no. 1.-S: 38-45.

14. Van Leer, B. (1979) "Towards the Ultimate Conservative Difference Scheme, V. A Second Order Sequel to Godunov's Method." J. Comput. Phys. 1979. V.32: 101-136.

15. Colella P. and Woodword P. (1984) The Piecewise Parabolic method for Gas-Dynamical Simulations.// J. Comp. Phys. 1984. V. 54: 174 - 202.

16. Abuziarov M.Kh., Bazhenov V.G., Kochetkov A.V (1987) About a new effective approach to improving the accuracy of the Godunov scheme, Applied problems of strength and ductility. Solution methods. All Interuniversity. Sb.Gork..1987.S. 43-49.

17. Abuziarov M.Kh., Bazhenov V.G., Kochetkov A.V. (1987) On the monotonization of the Godunov scheme of the second order of accuracy by introducing the scheme viscosity, PPPP. Research and optimization of structures: All-Union. interuniversity. Sat, Gorky: Publishing House of the GSU. 1987.S. 85-90. 
18. Abouziarov M., Aiso H., Takahashi T. (2004) An application of conservative scheme to structure problems // Series from Research Institute of Mathematics of Kyoto University. Mathematical Analysis in Fluid and Gas Dynamics. 2004, 1353: 192-201.

19. Abouziarov, H. Aiso (2004) An application of retroactive characteristic method to conservative scheme for structure problems (elastic-plastic flows). // Hyperbolic Problems, Theories, Numerics, Appli-cations. Tenth International Conference in Osaka. September 2004, Copiright 2006 by Yokohama Publishers, Inc. p. 223-230.

20. G. Miller, P. Colella (2001) A high-order Eulerian Godunov method for elastic - plastic flow in solids, J. Comput. Phys. 167(1): 131-176.

21. H. Miller (2004) An iterative Riemann solver for systems of hyperbolic conservation laws, with application to hyperelastic solid mechanics, J. Comput. Phys. 193(1): 198-225.

22. I. Menshov, R. Zakharov (2014) "Op the composite Riemann problem for multi-material fluid flows", Int. J. Numer. Meth. Fluids 76: 2: 109-127 crossref MathSciNet

23. Kochetkov A.V (1991) Application of the flow correction method to solving non-stationary problems of the theory of elasticity // Prikl. problems of strength and ductility. Solution methods. Gorky: GSU, 1991. S. 32-37.

24. Rozhdestvensky B.L., Yanenko N.N (1978) Systems of quasilinear equations and their application to gas dynamics. M .: Nauka, 1978.

25. Kukudzhanov V.N (2004) The splitting method of elastoplastic equations // Izv. RAS MTT. 2004. 1: 98-108.

26. K.M. Abuziarov, M.Kh. Abuziarov S.V. Zefirov (2014) Numerical method for determining explosive loads in Eulerian variables on spatial structures during detonation of solid explosives // Problems of strength and ductility: Interuniversity. Sat / Nizhny Novgorod. un-t 2014. Issue. 76 (4): 326-334.

27. Wilkins M.L (1967) Calculation of Elastoplastic Flows // Computational Methods in Hydrodynamics / M.: Mir, 1967, 212-263.

28. Zlatin N.A., Mishin G.I. (1974) Ballistic installations and their application in experimental studies. $\mathrm{M}$.: Nauka, 1974.

29. Abuziarov M.Kh., Krylov S.V., Tsvetkova E.V. (2013) Modeling of hydroelastic elastic interaction using the UPSGOD software package. Problems of strength and ductility. 2013. Issue. 75 (1): 25-32.

Copyright: (C2021 M.H.Abuziarov. This is an open-access article distributed under the terms of the Creative Commons Attribution License, which permits unrestricted use, distribution, and reproduction in anymedium, provided the original author and source are credited. 